\title{
Morphological and molecular identification of Fusarium spp. isolated from maize kernels in Java and Lombok, Indonesia
}

\author{
ANI WIDIASTUTI ${ }^{1, \bullet}$, MONICA LUCKY KARLINA ${ }^{1}$, KURNIA RITMA DHANTI ${ }^{2}$, YUFITA DWI CHINTA ${ }^{3}$, \\ TRI JOKO ${ }^{1}$, SURYANTI ${ }^{1}$, ARIF WIBOWO ${ }^{1}$ \\ ${ }^{1}$ Faculty of Agriculture, Universitas Gadjah Mada. J1. Flora No 1. Bulaksumur, Sleman 55281, Yogyakarta, Indonesia. Tel./fax.: +62-274-523926, \\ •email: aniwidiastuti@ugm.ac.id \\ ${ }^{2}$ Universitas Muhammadiyah Purwokerto. J1. Raya Dukuhwaluh, Kembaran, Banyumas 53182, Central Java, Indonesia \\ ${ }^{3}$ Biosphere Science Division, Agro-ecosystem course, Graduate School of Environmental Science, Hokkaido University. North 10 West 5, Sapporo, \\ Hokkaido 060-0810, Japan
}

Manuscript received: 27 December 2019. Revision accepted: 25 May 2020.

\begin{abstract}
Widiastuti A, Karlina ML, Dhanti KR, Chinta YD, Joko T, Suryanti, Wibowo A. 2020. Morphological and molecular identification of Fusarium spp. isolated from maize kernels in Java and Lombok, Indonesia. Biodiversitas 21: 2741-2750. Fungal contamination of maize is a serious problem in Indonesia. Fusarium spp. infect maize in the field will be continuing to contaminate in the post-harvest period even though disease symptomps are not always emerged. Some Fusarium spp. produced mycotoxins which are harmful to human and animal health. Aims of this research were to reveal the presence of Fusarium spp. from both symptomatic and unsymptomatic maize, and to identify them based on morphological characteristics and molecular analysis. Samples of maize were collected from maize cultivation areas in East Java (EJ), Central Java (CJ), West Java (WJ), Yogyakarta Special Province (DIY) and Lombok, West Nusa Tenggara. Fusarium spp. were isolated in a single spore method and cultured in potato dextrose agar (PDA) medium for morphological identification of macro-and microconidia. Molecular identification was conducted by PCR assay using species-specific primers. Furthermore, unidentified species were analyzed by DNA sequence. This research found four species of mycotoxigenic Fusarium isolated from maize based on molecular identification, which were Fusarium verticillioides (15 isolates), $F$. proliferatum (6 isolates), $F$. graminearum (1 isolate) and $F$. asiaticum (1 isolate). This research showed a novel report of $F$. asiaticum infection on maize kernel in Indonesia.
\end{abstract}

Keywords: Fusarium asiaticum, F. graminearum, F. proliferatum, F. verticiloides, identification, maize, mycotoxin

\section{INTRODUCTION}

Maize (Zea mays) is one of the most prominent crops used as human food directly and cattle and birds food source indirectly (Mostafa and Kazem 2011). In LatinAmerican and Africa, maize rank first, whether in Asia, it ranks third after rice and wheat (Mejia 2003), and it is the second important crop after rice in Indonesia. Maize is largely planted in several areas of Indonesia, including East Java, Central Java, Lampung, East Nusa Tenggara, West Nusa Tenggara, North Sumatera, South Sulawesi (Swastika et al. 2004).

Grain losses contribute to food insecurity and low farm incomes in the world (Komen et al. 2008). High relative humidity and grain water content during storage provide higher opportunity for infection of toxin producing fungi such as Fusarium spp on maize. F. verticilloides is reported to be one important pathogen on maize in Indonesia, both in field and storage (Pakki 2016). Fusarium species rapidly colonize the maize residues in soil and being established in maize seeds and grains. $F$. proliferatum and $F$. verticillioides species will remain in the residues of maize which are probably the main source of infection. Prevalence of infection by toxin producer fungi species play a prominent role in the existence of mycotoxic contaminations (Mostafa and Kazem 2011).
The genus Fusarium comprises more than 80 species of fungi, which can parasite plants, vertebrates, insects, humans, or even other fungi (Ivic et al. 2009). It is the most diverse group and harmful pathogen in food and feed grains, including maize. Fusarium species produce long, multicellular, canoe-shaped or banana-shaped macroconidia. These large asexual conidia are the defining morphological characteristic of the genus. Many species will also produce small, generally single-celled microconidia that range in shape from fusiform to oval to spherical. Additionally, some species produce thick-walled resistant chlamydospores important for long-term survival (Glenn 2007). In general, most of Fusarium spp. have a strong saprophytic ability and can develop on dead organic matter (Ivic et al. 2009).

In addition, species of Fusarium spp. can produce a wide range of secondary metabolites, many of which are a potential health hazards for humans and animals (Ivic et al. 2009). Several mycotoxins produced by Fusarium spp. are diacetoxyscirpenol, deoxynivalenol, nivalenol, T-2 toxin, zearalenone, fumonisins, fusarin $\mathrm{C}$, beauvericin, moniliformin, and fusaproliferin. These toxics disrupt the growth and development of animals and cause cancer in humans (Glenn 2007). The presence of those mycotoxins depends on strains of fungi, location and climate, breeding technique, plant susceptibility, and storage condition 
(Akadri 2012). For instance, Deoxynivalenol (DON) is one of the most common mycotoxins found in grains, including maize. It causes nausea, vomiting, and diarrhea; at lower doses, pigs and other farm animals exhibit weight loss and food refusal (Bennet and Klinch 2003).

In terms to understand the interaction between species Fusarium spp. and maize in the post-harvest period, identification of Fusarium spp. based on the morphology of culture is an essential fact. Moreover, sensitive and specific molecular analysis, such as species-specific polymerase chain reaction (PCR) assay, can enhance the identification data (Jurado et al. 2005). Therefore, basic research was conducted to identify Fusarium spp. from maize kernels both symptomatic and unsymtompatic to understand the fungal contamination in maize.

\section{MATERIALS AND METHODS}

\section{Samples collection}

Samples of maize were collected from fields and some are from storages. Samples collected from fields were from East Java Province (Nganjuk, Kediri), Central Java Province (Klaten, Purbalingga, Temanggung), West Java Province (Bandung, Bogor), Yogyakarta Special Province (Sleman), and from West Nusa Tenggara Province (West Lombok). Samples collected from storage were from Malang (East Java Province), Wonosobo (Central Java Province) and Bantul (Yogyakarta Special Province) (Figure 1). Each sample consists of 500 grams of kernels.

\section{Single spore isolation of Fusarium spp.}

Isolation of Fusarium spp. was conducted in potato dextrose agar (PDA) medium. Thirty kernels of maize were sterilized in $1 \%$ of sodium hypochlorite solution for 1 minute, washed twice with sterile water, and then dried in a laminar flow cabinet. Clean kernels were placed in PDA medium, 10 kernels per petri dish and incubated at $28^{\circ} \mathrm{C}$ of temperature for 7 days in dark condition. Fusarium fungi from kernels were sub-cultured in new PDA medium. The collected Fusarium were isolated by single spore method described by Alvindia et al. 2001. Spore suspension was streaked in water agar $2 \%$ medium and incubated during 12-18 h. After that, the single germinated-spore was taken and cultured in PDA medium.

\section{Morphological identification of Fusarium spp.}

Fusarium spp. was grown in PDA for identifying morphological colonies, pigmentation, size of macroconidia and microconidia. Identification was made according to Leslie and Summerell (2006).

\section{Molecular identification}

Isolates of Fusarium spp. were prepared in potato dextrose broth (PDB) medium by shaking in a low speed rotary shaker for 7 days at room temperature. Mycelia were collected, and then the DNA was extracted using DNA extraction kit (Nucleospin Plant II, Macherey-Nagel, Germany) as a procedure. DNA was amplified using PCR by species-specific primers as following in table 1 . The PCR was done in $20 \mu \mathrm{l}$ reaction volume containing $2.6 \mu \mathrm{l}$ of distilled water (DW), $10 \mu \mathrm{l}$ of $2 \times$ buffer, $4 \mu \mathrm{l}$ of dNTPs, $1 \mu \mathrm{l}$ of $5 \mu \mathrm{M}$ each primer, $0.4 \mu \mathrm{l}$ of the enzyme, and $1 \mu \mathrm{l}$ of each DNA extract. PCR was done by a thermal cycler (MyCycler, Bio-Rad Laboratories Pty. Ltd., Australia). Electrophoresis of PCR product is conducted in $0.8 \%$ agarose (Agarose H14 Takara, Takara, Japan) on buffer TBE with $100 \mathrm{~V}$ of voltage (Mupid-2Plus, Advance, Japan). Unidentified species of Fusarium spp. were sent to a sequencer company for the sequencing analysis. The results were analyzed using sequencing analysis program: Mega5, Bioedit and Genetyx.

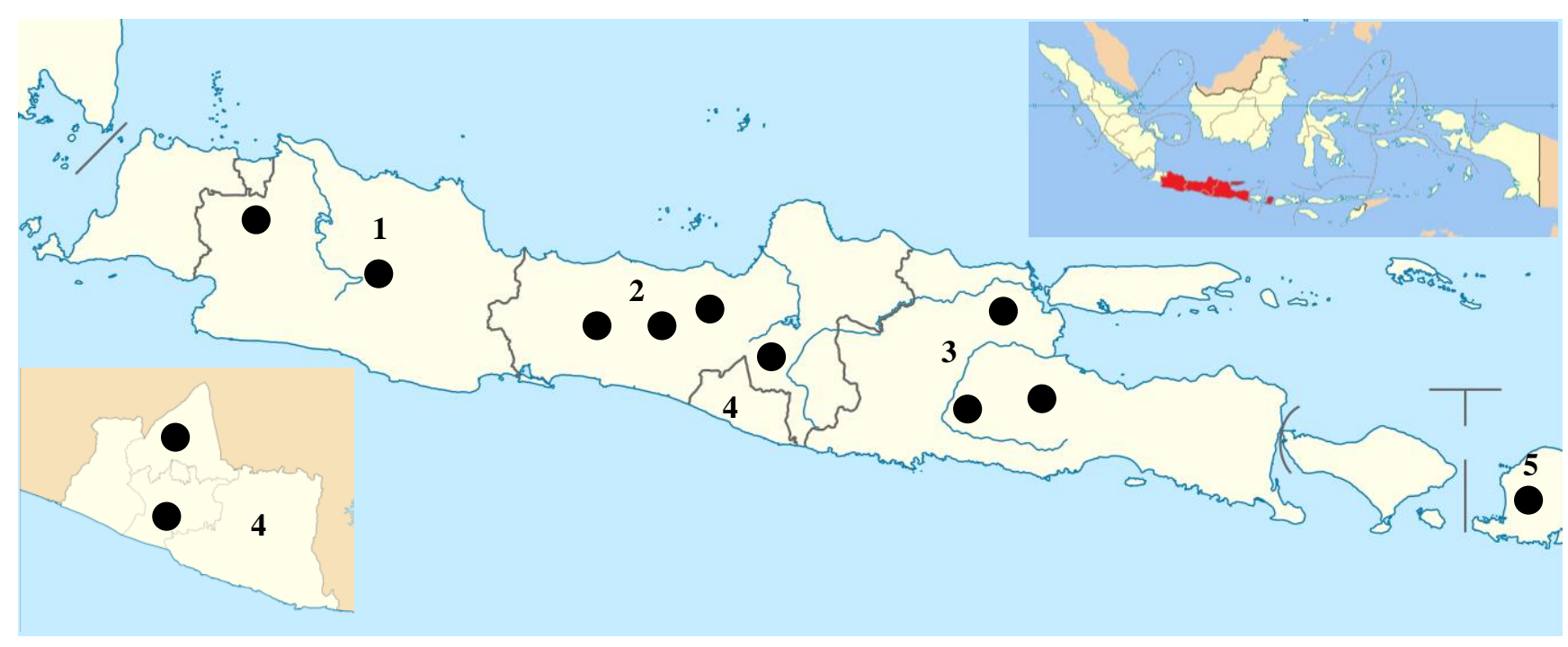

Figure 1. Map of maize samples collection in Java and Lombok Islands, Indonesia. 1. West Java Province (WJ); 2. Central Java Province (CJ); 3. East Java Province (EJ); 4. Yogyakarta Special Province (DIY); 5. West Nusa Tenggara Province (L); • Location of samples collection 
Table 1. Species-specific primers used for Fusarium spp. identification

\begin{tabular}{|c|c|c|c|c|c|}
\hline $\begin{array}{c}\text { Species of } \\
\text { Fusarium spp. }\end{array}$ & Primers & Primer sequence $(5 \rightarrow 3)$ & $\begin{array}{l}\text { Size } \\
(\text { bp) }\end{array}$ & PCR condition & Reference \\
\hline \multirow[t]{2}{*}{ F. avenaceum } & JIA-F & GCTAATTCTTAACTTACTAGGGGCC & \multirow[t]{2}{*}{$\sim 220$} & $95^{\circ} \mathrm{C} 3 \mathrm{~min} ; 95^{\circ} \mathrm{C} 30 \mathrm{~s}$ & \multirow[t]{2}{*}{ Turner, et al. (1998) } \\
\hline & JIA-R & CTGTAATAGGTTATTTACATGGGCG & & $\begin{array}{l}62^{\circ} \mathrm{C} 20 \mathrm{~s} ; 72^{\circ} \mathrm{C} 45 \mathrm{~s} ; 38 \\
\text { cycles; } 72^{\circ} \mathrm{C} 2 \mathrm{~min}\end{array}$ & \\
\hline \multirow[t]{2}{*}{ F. culmorum } & Fcu-F & GACTATCATTATGCTTGCGAGAG & \multirow[t]{2}{*}{$\sim 200$} & $94^{\circ} \mathrm{C} 85 \mathrm{~s} ; 95^{\circ} \mathrm{C} 35 \mathrm{~s}$ & \multirow[t]{2}{*}{ Jurado et al. (2005) } \\
\hline & Fgc-R & СТCТСАТАТАСССТCCG & & $\begin{array}{l}54^{\circ} \mathrm{C} 30 \mathrm{~s} ; 72^{\circ} \mathrm{C} 30 \mathrm{~s} ; 20 \\
\text { cycles } ; 72^{\circ} \mathrm{C} 5 \mathrm{~min}\end{array}$ & \\
\hline \multirow[t]{2}{*}{ F. graminearum } & Fgr-F & GTTGATGGGTAAAAGTGTG & \multirow[t]{2}{*}{$\sim 500$} & $94^{\circ} \mathrm{C} 85 \mathrm{~s} ; 95^{\circ} \mathrm{C} 35 \mathrm{~s}$ & \multirow{2}{*}{$\begin{array}{l}\text { Jurado et al. }(2006 \mathrm{a}, \mathrm{b}) \\
\text { Sampietro et al. }(2010)\end{array}$} \\
\hline & Fgc-R & СТCTCATATACССТCCG & & $\begin{array}{l}53^{\circ} \mathrm{C} 30 \mathrm{~s} ; 72^{\circ} \mathrm{C} 30 \mathrm{~s} ; 25 \\
\text { cycles } ; 72^{\circ} \mathrm{C} 5 \mathrm{~min}\end{array}$ & \\
\hline \multirow[t]{2}{*}{ F. proliferatum } & Fp3-F & CGGCCACCAGAGGATGTG & \multirow[t]{2}{*}{$\sim 230$} & $94^{\circ} \mathrm{C} 85 \mathrm{~s} ; 95^{\circ} \mathrm{C} 35 \mathrm{~s}$ & \multirow{2}{*}{$\begin{array}{l}\text { Jurado et al. (2006a,b); } \\
\text { Sampietro et al. (2010) }\end{array}$} \\
\hline & Fp4-R & CAACACGAATCGCTTCCTGAC & & $\begin{array}{l}65^{\circ} \mathrm{C} 30 \mathrm{~s} ; 72^{\circ} \mathrm{C} 30 \mathrm{~s} ; 25 \\
\text { cycles } ; 72^{\circ} \mathrm{C} 5 \mathrm{~min}\end{array}$ & \\
\hline \multirow[t]{2}{*}{ F. verticilliodes } & Fps-F & CGCACGTATAGATGGACAAG & \multirow[t]{2}{*}{$\sim 700$} & $94^{\circ} \mathrm{C} 85 \mathrm{~s} ; 95^{\circ} \mathrm{C} 35 \mathrm{~s}$ & \multirow{2}{*}{$\begin{array}{l}\text { Jurado et al. (2006a,b) } \\
\text { Sampietro et al. (2010) }\end{array}$} \\
\hline & Vert2 & CACCCGCAGCAATCCATCAG & & $\begin{array}{l}65^{\circ} \mathrm{C} 30 \mathrm{~s} ; 72^{\circ} \mathrm{C} 30 \mathrm{~s} ; 25 \\
\text { cycles } ; 72^{\circ} \mathrm{C} 5 \mathrm{~min}\end{array}$ & \\
\hline \multirow[t]{2}{*}{ F. subglutinans } & SUB1 & CTGTCGCTAACCTCTTTATCCA & \multirow[t]{2}{*}{$\sim 600$} & $94^{\circ} \mathrm{C} 5 \mathrm{~min} ; 94^{\circ} \mathrm{C} 90 \mathrm{~s}$ & \multirow[t]{2}{*}{ Mulè et al. (2004) } \\
\hline & SUB2 & CAGTATGGACGTTGGTATTATATCTAA & & $\begin{array}{l}56^{\circ} \mathrm{C} 90 \mathrm{~s} ; 72^{\circ} \mathrm{C} 2 \mathrm{~min} ; 30 \\
\text { cycles; } 72^{\circ} \mathrm{C} 10 \mathrm{~min}\end{array}$ & \\
\hline
\end{tabular}

\section{RESULTS AND DISCUSSION}

This research was a basic research to collect and identify Fusarium spp. infecting maize kernels from some centers of maize production in Java. to study the species name of collected Fusarium, molecular analysis instead of morphological observation was needed because their morphological characters varied very widely. In this research, morphological observation is used to confirm the molecular identification. The molecular identification was conducted based on universal primer mitochondrial small ribosomal subunit (MtSSu) with DNA target was 690 bp and all isolates showed the DNA bands (Aoki 2009).

Fusarium spp. have very wide range in size of their conidia, therefore difficult to find the species based on their morphological characters. Data of macro-and microconidia size were shown in Table 2. Only isolates M1 and W3 have similar characteristic that they did not produce microconidia and posses strong red pigment. One species of pathogenic Fusarium on maize that do not produce microconidia is $F$. graminearum (Leslie and Summerell 2006). Based on characteristic morphology and red pigment production, they referred to the mentioned species however molecular analysis is needed to confirm. $F$. graminearum complex is a group of Fusarium spp. that contains of several related species, such as $F$. graminearum, $F$. culmorum, $F$. cerealis, $F$. vorosii, and $F$. asiaticum (Aoki 2009).

Based on identification of their species-specific primers, the collected Fusarium spp. are identified as shown in Table 3: F. verticillioides were collected from Bantul [DIY], Purbalingga [CJ], Wonosobo [CJ], Klaten $[\mathrm{CJ}]$, Temanggung [CJ], Kediri [EJ], Nganjuk [EJ], Malang $[\mathrm{EJ}]$, Gerung $[\mathrm{L}]$, Bandung [WJ], Bogor [WJ]. $F$. proliferatum were collected from Bantul [DIY], Kediri [EJ] and Purbalingga $[\mathrm{CJ}]$. F. graminearum was isolated from Wonosobo [CJ]. Two isolates of $F$. verticilloides and $F$. proliferatum which grew together in media and two isolates from Sleman [DIY] need further identification.
Molecular data and morphological characters based on their species are elaborated below.

\section{Fusarium verticillioides}

Fifteen isolates of $F$. verticilloides were isolated from maize kernels and identified using specific primers and morphological characters observation as below (Figure 2). They were mainly found from unsimptomatic maize kernels showed that maize were contaminated by the fungi without any symptom. The fungal status becomes significant because genes related to fumonisin, deoxynivalenol and zearalenon were detected in those $F$. verticilloides (Dhanti et al. 2017).

Table 3. Fusarium spp. based on species-specific primers identification

\begin{tabular}{lll}
\hline Species & Isolate ID & Origin \\
\hline F. verticillioides & BK & Bantul \\
& Na & Nganjuk \\
& Nb.1 & Nganjuk \\
& Nb.2 & Nganjuk \\
& P4 & Purbalingga \\
& P5 & Purbalingga \\
& W1 & Wonosobo \\
& K & Klaten \\
& TG1 & Temanggung \\
& TG2 & Temanggung \\
& KD2 & Kediri \\
& GR2 & Gerung \\
& M2 & Malang \\
& BG & Bandung \\
& BR & Bogor \\
& UGM2 & Bantul \\
& UGM3 & Bantul \\
& UGM4 & Bantul \\
& P3 & Purbalingga \\
& BTb & Bantul \\
& KD2 & Kediri \\
& W3 & Wonosobo \\
& &
\end{tabular}

Note: *confirmed in specific primer after sequencing result. 
Fusarium verticillioides showed pigment variation in PDA medium, dominantly in plum and violet. Mycelia are white and have flat growth (Figure 3). Microscopic observation showed that their isolates have conidiogenous cell in monophialid and often in long chains (Figure 4) as written in Leslie and Summerell (2006).

DNA extraction of TG2 isolate did not show any band in species-specific primers analysis. However, based on phylogenetic tree analysis, this isolate showed closely relate to $F$. verticillioides using Histone H3 gene (Aoki 2009), with homology 99.557\% (Figure 5 and Tab. 4). After confirmed with some DNA dilution using $F$. verticillioides-specific primer, slight band appeared. It possibly due to the amount of DNA quantity or quality that need to be improved.

Table 4. Homology data of TG2 isolate

\begin{tabular}{lll}
\hline $\begin{array}{l}\text { Isolate } \\
\text { ID }\end{array}$ & Homology species & $\begin{array}{l}\text { Homology } \\
\text { value }\end{array}$ \\
\hline TG2 & F. nygamai FRCM-7492 & $95.344 \%$ \\
& F. pseudocircinatum NRRL 53570 & $94.235 \%$ \\
& F. ramigenum NRRL 25208 & $94.457 \%$ \\
& F. verticillioides FRC M-3125 & $99.557 \%$ \\
\hline
\end{tabular}

Table 2. Colony color, size and septa's number of macroconidia and microconidia from collected Fusarium spp.

\begin{tabular}{|c|c|c|c|c|}
\hline Isolate ID & $\begin{array}{l}\text { Origin } \\
\text { (Province) }\end{array}$ & Macroconidia* (septa) & Microconidia* (septa) & Colony color \\
\hline M1 & Malang (EJ) & $34.7 \times 3.6 \mu \mathrm{m} \sim(3-8)$ & Absent & Red \\
\hline $\mathrm{Bk}$ & Bantul (DIY) & $11.82 \times 2.95 \mu \mathrm{m} \sim(2-4)$ & $4.27 \times 2.03 \mu \mathrm{m} \sim(0-1)$ & Plum \\
\hline $\mathrm{Na}$ & Nganjuk (EJ) & $11.41 \times 2.57 \mu \mathrm{m} \sim(2-3)$ & $4.63 \times 1.89 \mu \mathrm{m} \sim(0)$ & Plum \\
\hline $\mathrm{Nb} .1$ & Nganjuk (EJ) & $14.5 \times 2.9 \mu \mathrm{m} \sim(3-5)$ & $5.1 \times 2.04 \mu \mathrm{m} \sim(0)$ & Violet \\
\hline $\mathrm{Nb} .2$ & Nganjuk (EJ) & $37 \times 3.13 \mu \mathrm{m} \sim(3-9)$ & $4.82 \times 1.99 \mu \mathrm{m} \sim(0-1)$ & Brown \\
\hline W3 & Wonosobo (CJ) & $34.8 \times 3.3 \mu \mathrm{m} \sim(4-5)$ & Absent & Red \\
\hline UGM2 & Bantul (DIY) & $11.3 \times 2.89 \mu \mathrm{m} \sim(2-5)$ & $4.03 \times 1.96 \mu \mathrm{m} \sim(0-1)$ & Plum \\
\hline UGM3 & Bantul (DIY) & $13.3 \times 2.6 \mu \mathrm{m} \sim(2-3)$ & $5.4 \times 1.7 \mu \mathrm{m} \sim(0-1)$ & Misty-rose \\
\hline UGM4 & Bantul (DIY) & $34.25 \times 2.66 \mu \mathrm{m} \sim(5)$ & $6.9 \times 2.05 \mu \mathrm{m} \sim(0-1)$ & Violet \\
\hline P1 & Purbalingga (CJ) & $12.3 \times 3.2 \mu \mathrm{m} \sim(2-4)$ & $7.52 \times 1.9 \mu \mathrm{m} \sim(0)$ & Plum \\
\hline $\mathrm{P} 2$ & Purbalingga (CJ) & $11.8 \times 2.7 \mu \mathrm{m} \sim(2-7)$ & $4.4 \times 1.7 \mu \mathrm{m} \sim(0-1)$ & Violet \\
\hline P3 & Purbalingga (CJ) & $25.6 \times 2.5 \mu \mathrm{m} \sim(3-7)$ & $5.5 \times 2.2 \mu \mathrm{m} \sim(0-1)$ & Violet \\
\hline $\mathrm{P} 4$ & Purbalingga (CJ) & $30.08 \times 3.01 \mu \mathrm{m} \sim(3-5)$ & $5.32 \times 2.07 \mu \mathrm{m} \sim(0-1)$ & Plum \\
\hline P5 & Purbalingga (CJ) & $12.3 \times 3.3 \mu \mathrm{m} \sim(2-5)$ & $4.9 \times 2.3 \mu \mathrm{m} \sim(0-1)$ & Violet \\
\hline W1 & Wonosobo (CJ) & $36.41 \times 2.92 \mu \mathrm{m} \sim(3-7)$ & $5.62 \times 1.99 \mu \mathrm{m} \sim(0-2)$ & Moccasin \\
\hline $\mathrm{K}$ & Klaten (CJ) & $12 \times 3 \mu \mathrm{m} \sim(3-4)$ & $5.47 \times 2.3 \mu \mathrm{m} \sim(0-1)$ & Thistle \\
\hline TG1 & Temanggung (CJ) & $8.2 \times 2.22 \mu \mathrm{m} \sim(1-3)$ & $3.43 \times 1.83 \mu \mathrm{m} \sim(0-1)$ & Violet \\
\hline TG2 & Temanggung (CJ) & $12.7 \times 3.2 \mu \mathrm{m} \sim(3-5)$ & $5.4 \times 2.2 \mu \mathrm{m} \sim(0-1)$ & Brown \\
\hline MD2 & Sleman (DIY) & $10.4 \times 3.1 \mu \mathrm{m} \sim(2)$ & $4.1 \times 1.9 \mu \mathrm{m} \sim(0)$ & Violet \\
\hline MD3 & Sleman (DIY) & $38 \times 2.49 \mu \mathrm{m} \sim(5)$ & $7.52 \times 2.1 \mu \mathrm{m} \sim(0-1)$ & Chocholate \\
\hline ВТа & Bantul (DIY) & $10.5 \times 1.9 \mu \mathrm{m} \sim(3-5)$ & $4.9 \times 1.6 \mu \mathrm{m} \sim(0-1)$ & Brown \\
\hline $\mathrm{BTb}$ & Bantul (DIY) & $11.5 \times 2.56 \mu \mathrm{m} \sim(3)$ & $4.5 \times 1.7 \mu \mathrm{m} \sim(0)$ & Thistle \\
\hline KD1 & Kediri (EJ) & $9.6 \times 2.7 \mu \mathrm{m} \sim(3)$ & $4.0 \times 2.0 \mu \mathrm{m} \sim(0)$ & Yellow \\
\hline KD2 & Kediri (EJ) & $12.1 \times 2.7 \mu \mathrm{m} \sim(3-4)$ & $4.5 \times 1.81 \mu \mathrm{m} \sim(0)$ & Chocholate \\
\hline GR2 & Gerung (L) & $13.6 \times 2.8 \mu \mathrm{m} \sim(4-5)$ & $5.1 \times 2.1 \mu \mathrm{m} \sim(0)$ & Violet \\
\hline M2 & Malang (EJ) & $13.16 \times 2.47 \mu \mathrm{m} \sim(2-6)$ & $4.48 \times 1.72 \mu \mathrm{m} \sim(0-1)$ & Violet \\
\hline BG & Bandung (WJ) & $11.3 \times 2.2 \mu \mathrm{m} \sim(4)$ & $4.4 \times 1.9 \mu \mathrm{m} \sim(0)$ & Violet \\
\hline $\mathrm{BR}$ & Bogor (WJ) & $12.4 \times 3.1 \mu \mathrm{m} \sim(3-5)$ & $5.4 \times 2.1 \mu \mathrm{m} \sim(0)$ & Plum \\
\hline
\end{tabular}

Note: *Size of macro-and microconidia were counted from 100 conidia of each
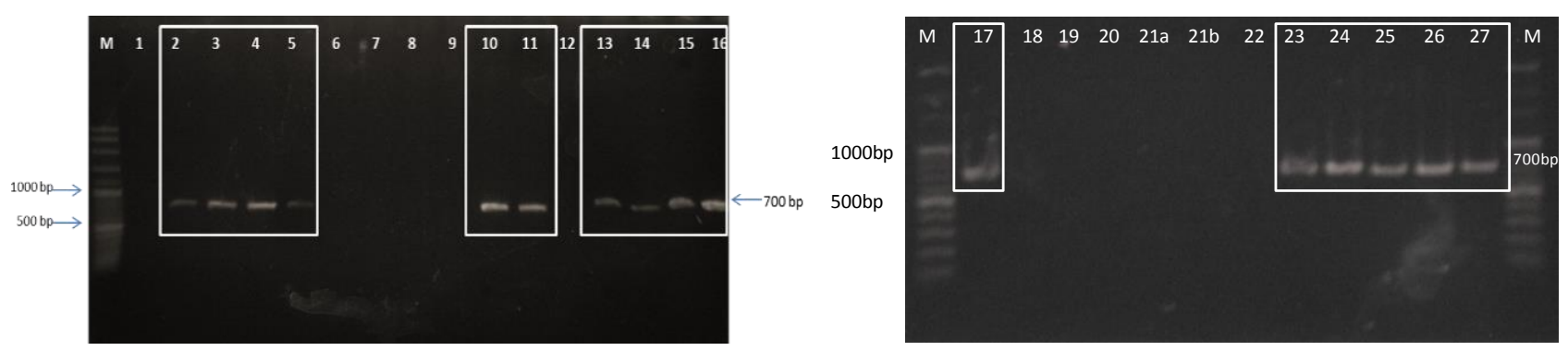

Figure 2. DNA band of Fusarium spp. using species specific primer for F. verticillioides. Sample no 2. Bk: Bantul (DIY); 3. Na: Nganjuk (EJ); 4. Nb.1: Nganjuk (EJ); 5. Nb2: Nganjuk (EJ); 10. P1*: Purbalingga (CJ); 11. P2*: Purbalingga (CJ); 13. P4: Purbalingga (CJ); 14. P5: Purbalingga (CJ); 15. W1: Wonosobo (CJ); 16. K: Klaten (CJ); 17. TG1: Temanggung (CJ); 23. KD2: Kediri (EJ); 24. GR2: Gerung (L); 25. M2: Malang (EJ); 26. BG: Bandung (WJ); 27. BR: Bogor (WJ). *Showed that isolates were still in a mixture with other species 


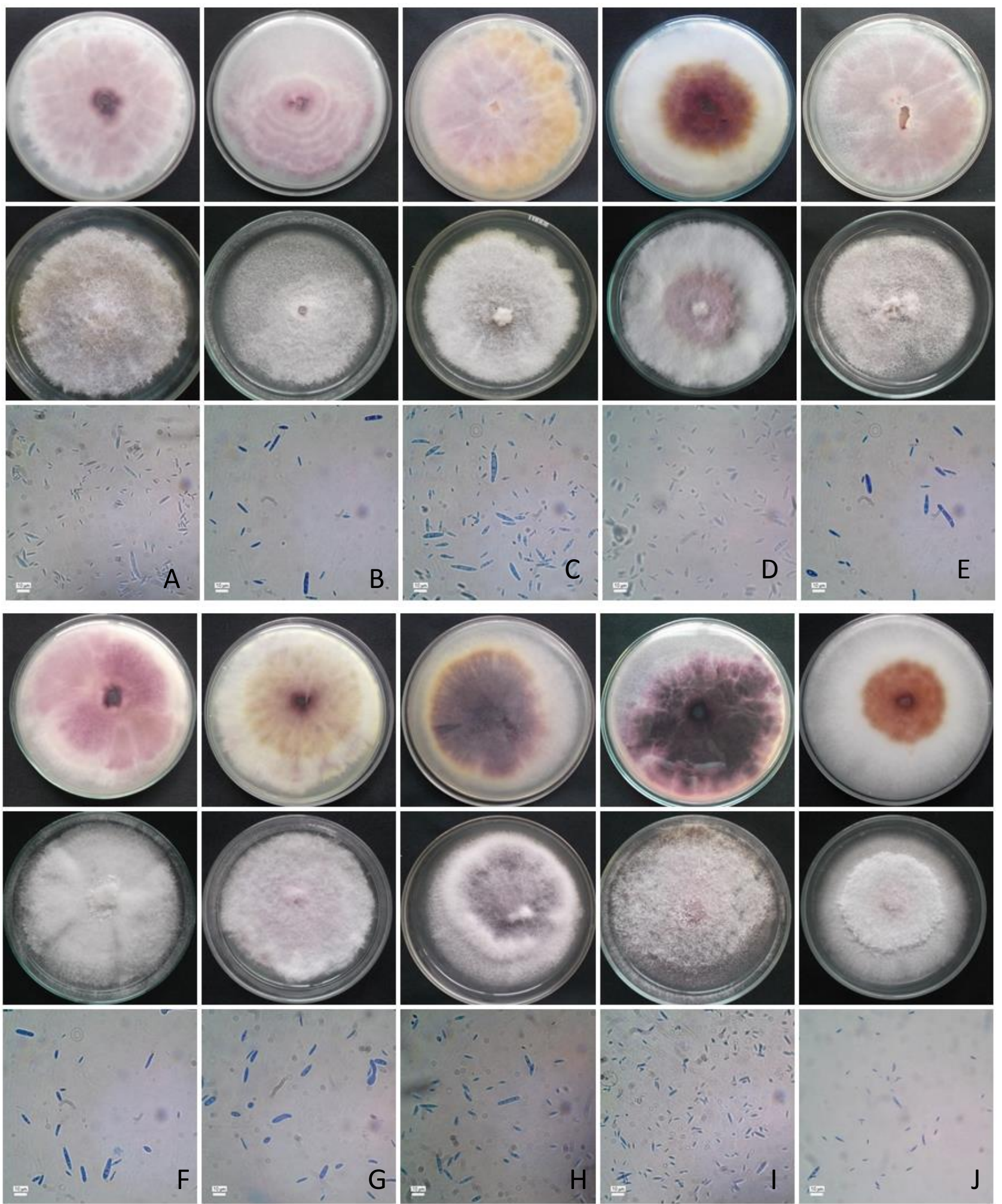



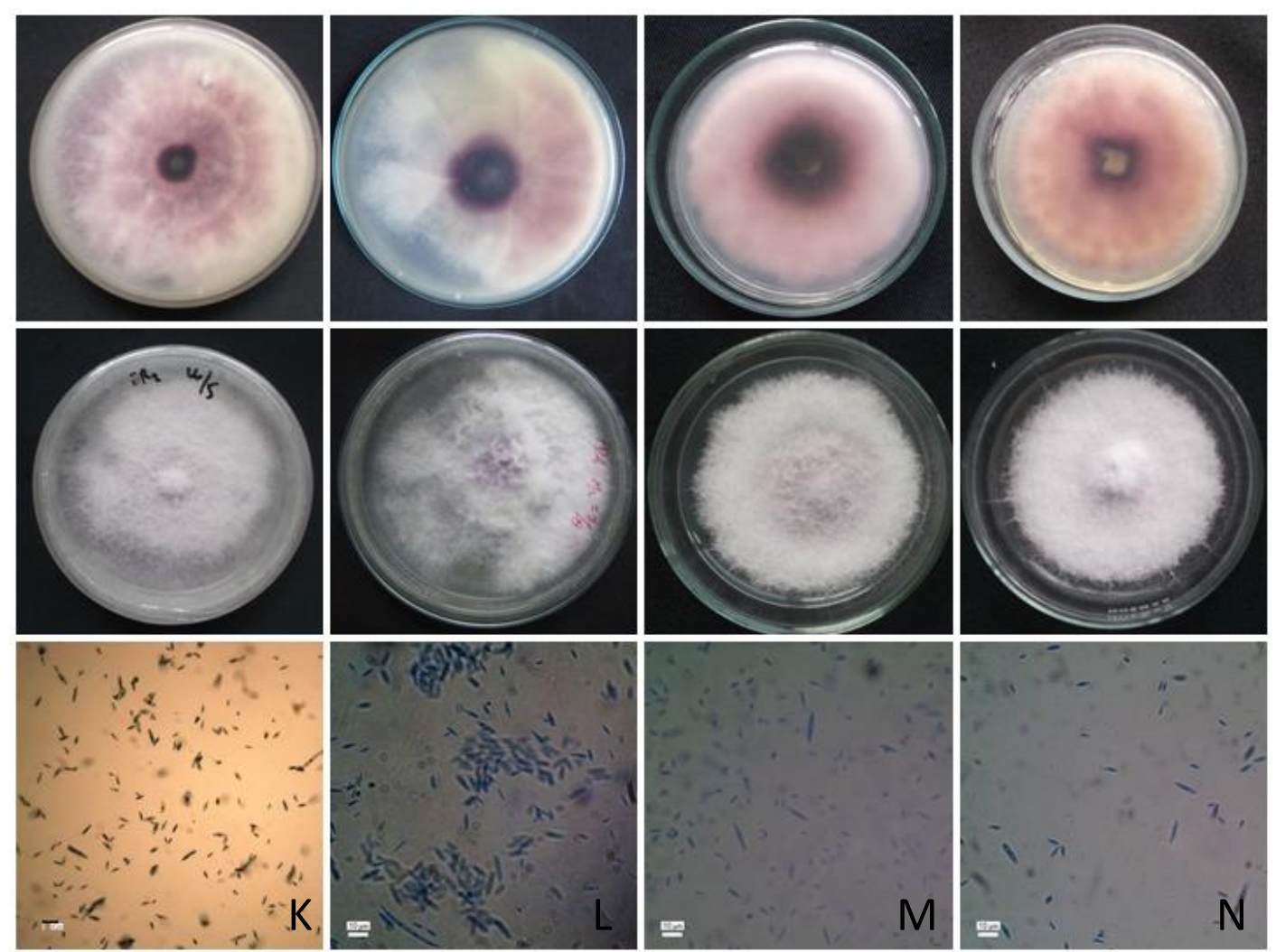

Figure 3. Culture and conidia of F. verticillioides in PDA medium. Bk: Bantul (DIY); B. Na: Nganjuk (EJ); C. Nb.1: Nganjuk (EJ); D. Nb2: Nganjuk (EJ); E. P4: Purbalingga (CJ); F. P5: Purbalingga (CJ); G. W1: Wonosobo (CJ); H. K: Klaten (CJ); I. TG1: Temanggung (CJ); J. KD2: Kediri (EJ); K. GR2: Gerung (L); L. M2: Malang (EJ); M. BG: Bandung (WJ); N. BR: Bogor (WJ)
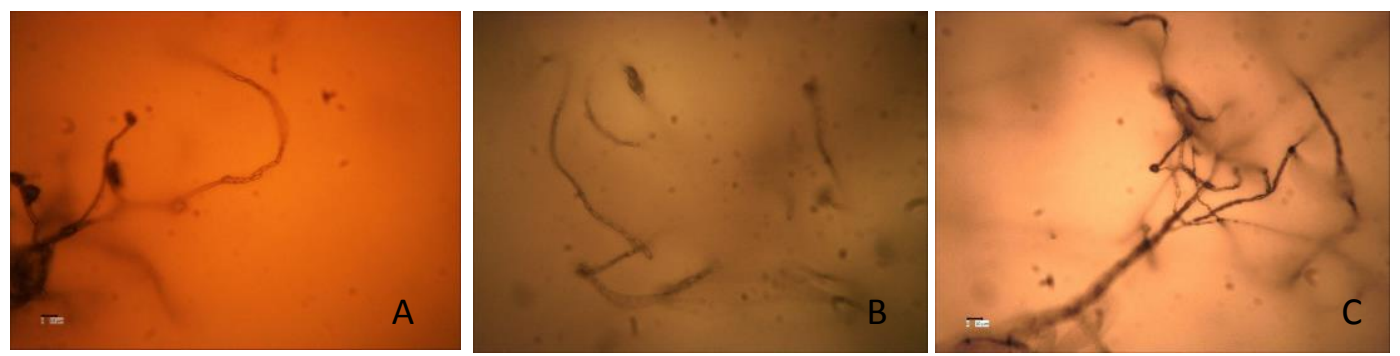

Figure 4. Conidiogenous cell of F. verticillioides. A. GR2: Gerung (L); B. TG1: Temanggung (CJ); C. BR: Bogor (WJ)

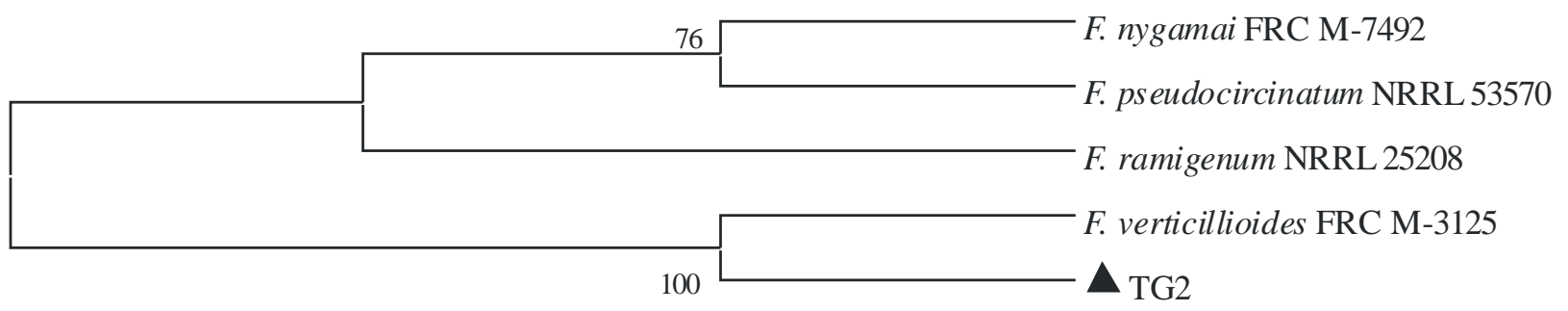

Figure 5. Phylogenetic tree of TG2 isolate using Histone H3 gene 

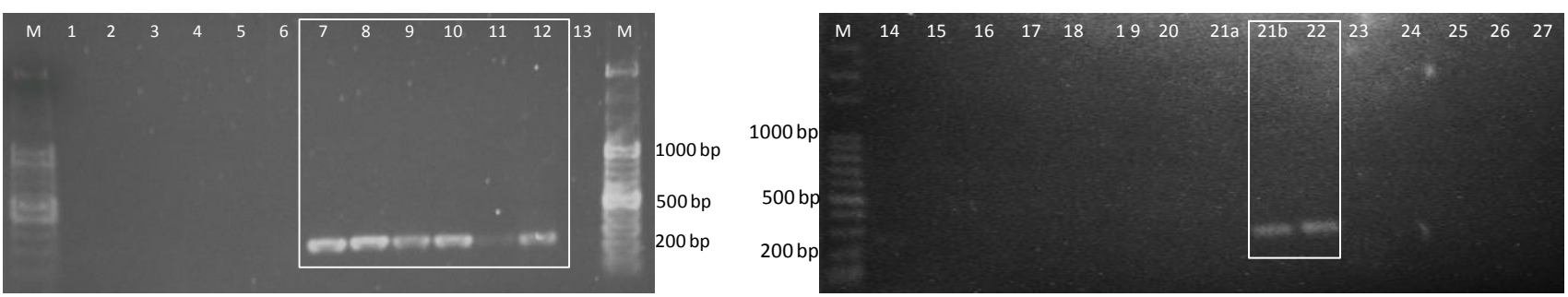

Figure 6. DNA band of Fusarium spp. using species specific primer for F. proliferatum. Sample no 7. UGM2: Bantul (DIY); 8.UGM3: Bantul (DIY); 9.UGM4: Bantul (DIY); 10. P1*: Purbalingga (CJ); 11.P2*: Purbalingga (CJ); 12.P3: Purbalingga (CJ); 21.b. BTb: Bantul (DIY); 22. KD1: Kediri (EJ). *Showed that isolates were still in a mixture with other species
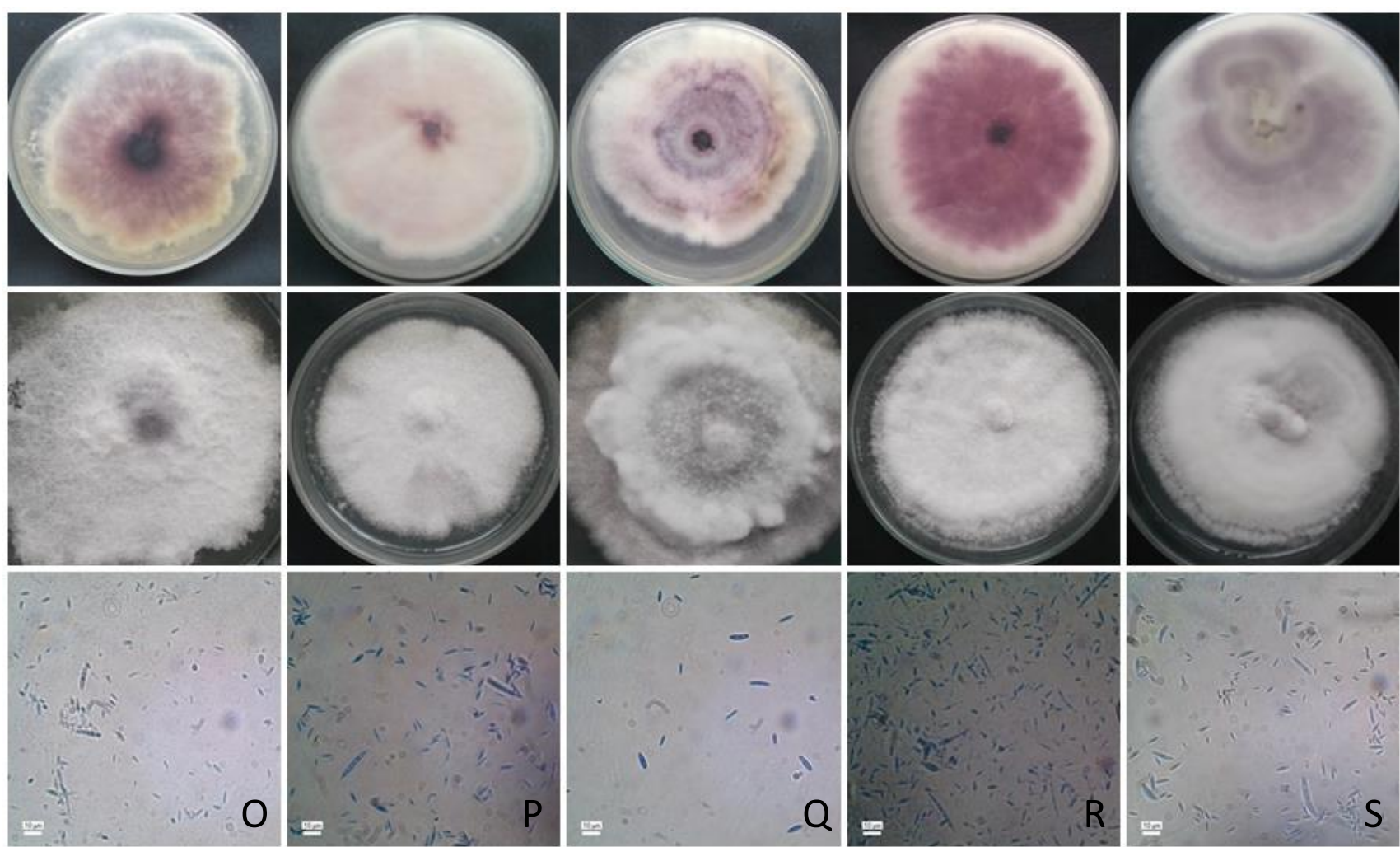

Figure 7. Culture and conidia of F. proliferatum in PDA medium. O. UGM2: Bantul (DIY); P. UGM3: Bantul (DIY); Q. UGM4: Bantul (DIY); R. P3: Purbalingga (CJ); S. BTb: Bantul (DIY).
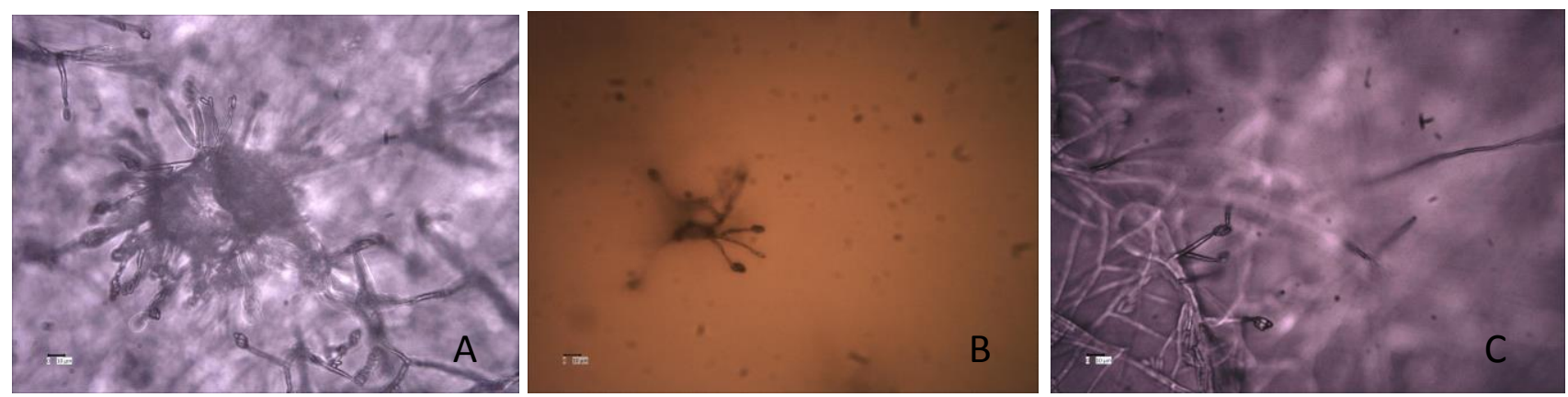

Figure 8. Conidiogenous cell of Fusarium proliferatum. A. UGM4: Bantul (DIY); B. P3: Purbalingga (CJ); C. BTb: Bantul (DIY) 


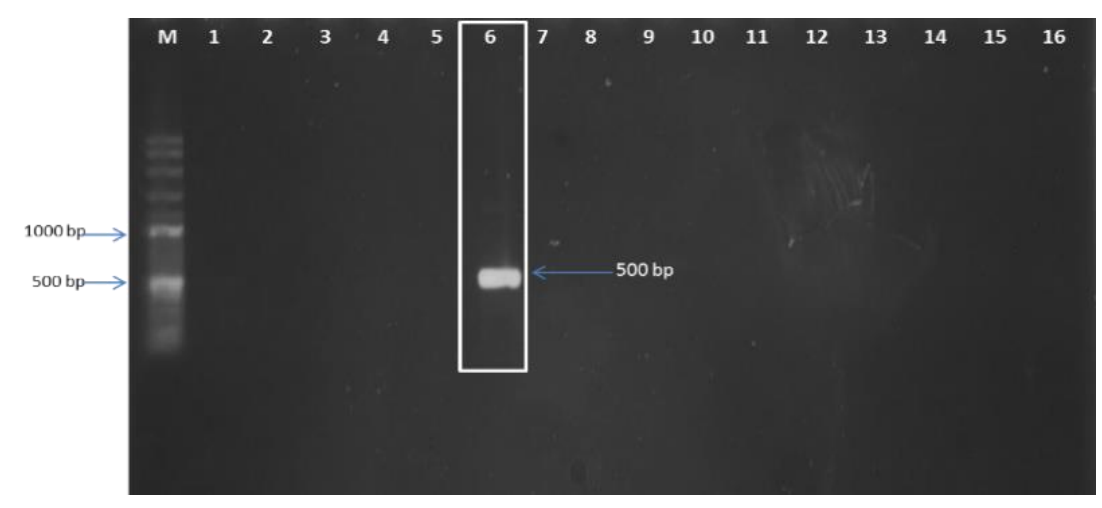

Figure 9. DNA band of Fusarium spp. using species specific primer for F. graminearum. Sample no 6. Is W3: Wonosobo (CJ)
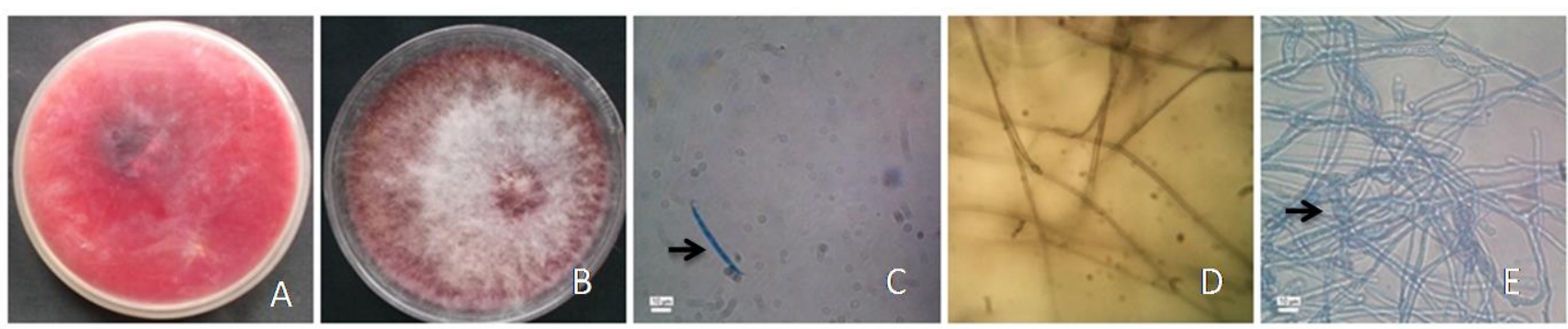

Figure 10. Culture and microscopic observation of Fusarium graminearum isolate W3 from Wonosobo. Lower part of isolate W3 in PDA medium; B. Upper part of isolate W3 in PDA medium. C. macroconidia (arrow); D. Mycelia without conidiogenous cell; E. Clamidospores (arrow)

\section{Fusarium proliferatum}

Six isolates of $F$. proliferatum were isolated from maize kernels mainly from unsymtompatic maize kernels. Result of molecular identification and morphological observation were showed below (Figure 6).

Pigment of $F$. proliferatum in PDA is also dominant in violet color, therefore difficult to be distinguished from $F$. verticillioides based on pigment production. However, the result showed that $F$. proliferatum often produced aerial, cotton-like mycelia (Figure 7). Conidiogenous cell usually produced in moderate length with mono-and polyphialid and microconidia in agregates (Figure 8) (Leslie and Sumerrell 2006).

In maize field, $F$. verticillioides is often being found together with $F$. proliferatum. They cause Fusarium ear rot (Rahjoo et al. 2008; Izzati et al. 2011; Darnetty and Saleh 2013). Both $F$. verticillioides and $F$. proliferatum are included in Giberella fujikuroi species complex, which possibly produce mycotoxins such as Fumonisin, Moniliformin and Beauvericin (Vincelli and Parker 2002; Tančić et al. 2012; Darnetty and Saleh 2013). Results in this research support those reports that $F$. verticillioides and $F$. proliferatum, single or together both of them, was dominantly isolated from the maize kernels.

\section{Fusarium graminearum species complex}

Based on specific primer identification and morphological character, one isolate was known as $F$. graminearum and another as $F$. asiaticum which its observation was showed below. F. graminearum isolate was confirmed by specific primer identification (Figure 9).
$F$. asiaticum were isolated from symptomatic ear rot of maize kernels which the kernel became pinkish due to fungal mycelia. To our best knowledge, this is the first report of $F$. asiaticum isolated from maize kernels in Indonesia. Genes related to deoxynivalenol and zearalenon were detected in $F$. graminearum and $F$. asiaticum (Dhanti et al. 2017) showed that these are important fungi have to be managed.

Fusarium graminearum produced red pigment in PDA medium, macroconidia and chlamidospores (Figure 10). There are no conidiogenos cells produced in mycelia. $F$. graminearum [teleomorph: Giberella zeae] is an important pathogen fungi caused Fusarium head blight (FHB) in wheat (Mansour et al. 2012; Muckle 2013). In maize, $F$. graminearum is an important pathogen that causes seed rot and seedling blight as well as root rot, stalk rot and ear rot.

One isolate from Malang [M1] has similar character in producing red pigment and the absence of microconidia and conidiogenos cells (Figure 11), however the DNA band was not found in the species-specific primer of $F$. graminearum or $F$. culmorum. Based on phylogenetic tree analysis, M1 isolate was closely relate to $F$. asiaticum using mitochondrial small ribosomal subunit (mtSSU) gene with homology value 100\% (Figure 12; Tab. 5). Aoki (2009) explained that $F$. graminearum and $F$. asiaticum were included in a group as $F$. graminearum species complex (FGSC). This complex species could produce three different types of mycotoxins, namely Deoxynivalenol (DON), Nivalenol (NIV) and Zearalenone (ZEA) (Vincelli and Parker 2002). 

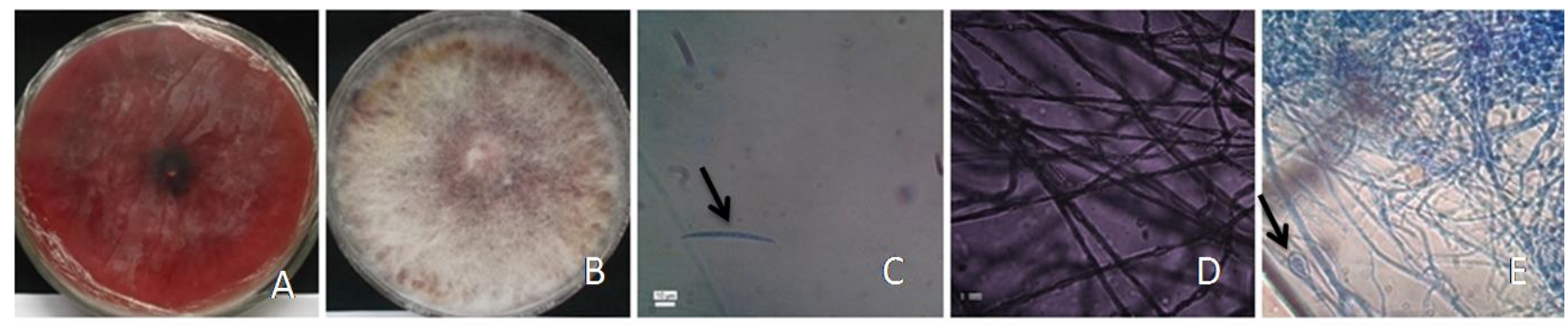

Figure 11. Culture and microscopic observation of isolate M1 from Malang. Lower part of isolate M1 in PDA medium; B. Upper part of isolate M1 in PDA medium. C. macroconidia (arrow); D. Mycelia without conidiogenous cell; E. Clamidospores (arrow)

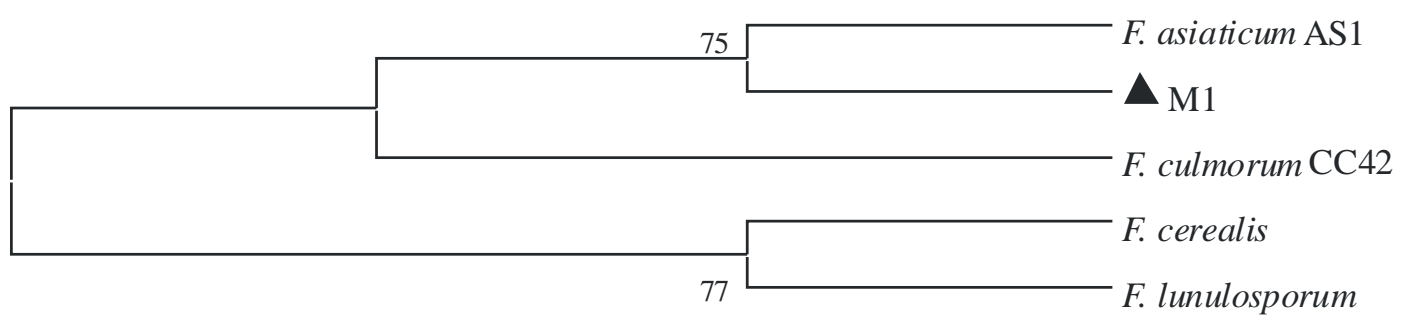

Figure 12. Phylogenetic tree of M1 isolate using MtSSU gene

Table 5. Homology data of M1 isolate

\begin{tabular}{llc}
\hline Isolate ID & Homology species & Homology value \\
\hline M1 & F. asiaticum AS1 & $100.000 \%$ \\
& F. cerealis & $98.452 \%$ \\
& F. culmorum CC42 & $98.762 \%$ \\
& F. lunulosporum & $98.452 \%$ \\
\hline
\end{tabular}

Up to date we have not found any other information of $F$. asiaticum on maize in Indonesia, therefore this result provided significant communication as an emerging pathogen in Indonesian maize. Due to advance increasing of fungal taxonomic and whole genome research, Walkowiak et al. (2016) found that whole genomics of $F$. asiaticum was closely related to $F$. graminearum. They were assumed to encode similar proteins employ in secondary metabolism and other functions consort with infection (Walwokiak et al. 2016), therefore they have similar characters such as in morphological characters and toxin production (Dhanti 2017). F. asiaticum was firstly reported as an emerging pathogen in postharvest asparagus in China (Zhu et al. 2013) and first reported to cause leaf spot in ornamental plant Bletia striata (Li et al. 2018). In maize, F. asiaticum was first reported as a pathogen caused ear rot in Japan (Kawakami et al. 2015) and one of major pathogens causing ear and kernel rot in China (Zhou et al. 2018). The important status of $F$. asiaticum both as a pathogen and mycotoxigenic fungi on maize should put it in the priority to research for food safety management in Indonesia.
Overall, 23 isolates of mycotoxigenic Fusarium were confirmed based on molecular identification. $F$. verticillioides (15 isolates) were collected from Bantul [DIY], Purbalingga [CJ], Wonosobo [CJ], Klaten [CJ], Temanggung [CJ], Kediri [EJ], Nganjuk [EJ], Malang [EJ], Gerung [L], Bandung [WJ], Bogor [WJ]. F. proliferatum (6 isolates) were collected from Bantul [DIY], Kediri [EJ] and Purbalingga [CJ], F. graminearum (1 isolate) was isolated from Wonosobo [CJ], and one species which was closely related to $F$. asiaticum (1 isolate) using MtSSU gene, isolated from Malang [EJ]. This research showed that molecular identification is important to recognize the species of Fusarium spp. based on the recent taxonomy science. Fusarium ear rot or ear blight is one of important diseases in maize need to be managed regarding the food safety issue as the Fusarium contamination did not always show the disease symptomp on maize kernels. To or best knowledge, it also reported novel finding of one important mycotoxigenic Fusarium which is $F$. asiaticum infection on maize kernels in Indonesia. This information gave great contribution in maize pre-and post-harvest handling as well as to understand the distribution of Fusarium spp. in Indonesia for disease management. As those Fusarium spp. were mycotoxigenic fungi, this report will be contributing greatly to the updated status of Fusarium spp. in Indonesia and globally.

\section{ACKNOWLEDGEMENTS}

Authors expressed sincere gratitude to Indonesian Toray Science Foundation which supported this research 
by STRG 20, 2014; and to Prof. Tatsuo Sato from College of Agriculture, Ibaraki University, Japan for providing MtSSU and H3 primers. This manuscript is a compilation report of a part of master thesis by Kurnia Ritma Dhanti entitled Molecular identification and detection of mycotoxin related gene of Fusarium spp. from maize kernels (2016) and undergraduate thesis by Monica Lucky Karlina entitled Morphological Characteristics of Fusarium spp. on Post Harvest Maize Kernels (2015) under supervision of first author and team. The authors declare that they have no conflict of interest.

\section{REFERENCES}

Alkadri D. 2012. Fusarium Species Responsible for Mycotoxin Production in Wheat Crop: Involvement in Food Safety. [Dissertation]. Department of Agroenvironmental Sciences and Technologies, Faculty of Agriculture, Bologna University, Italy.

Alvindia DG, Kobayashi T, Aoki T. 2001. Identification of fungi isolated from post harvest diseases of nonchemical bananas imported from The Phillipines. J Agr Sci Tokyo Univ Agric 45: 253-270.

Aoki T. 2009. Taxonomic System of The Genus Fusarium. Microbiol Cult Coll 25 (1): 1-12.

Bennet JW, Klinch M. 2003. Mycotoxins. Clinical Mycrobiol Rev 16 (3): 497-516.

Darnetty E, Salleh B. 2013. Toxigenicity of Fusarium species in Giberella fujikuroi species complex (GFSC) associated with stalk and ear rot disease of corn. J Plant Pathol 2 (3): 147-154.

Dhanti KR, Widiastuti A, Joko T. 2017. Detection of mycotoxin-related genes in Fusarium spp. isolated from maize kernels in Indonesia. Proceeding of 1st Internat. Conf. On Trop. Agric. Springer, New York. DOI 10.1007/978-3-319-60363-6.

Glenn AE. 2007. Mycotoxigenic Fusarium species in animal feed. Anim Feed Sci Technol 137: 213-240.

Ivic D, Domijan A, Peraica M et al. 2009. Fusarium spp. contamination of wheat, maize, soybean, and pea in Croatia. ArhHigRadaToksikol 60 435-442.

Izzati MZNA, Azmi AR, Nordahliawate MSS, Norazlina J. 2011. Contribution to the knowledge of diversity of Fusarium associated with maize in Malaysia. Plant Protect Sci 47 (1): 20-24

Jurado M, Va'zquez C, Callejas C, Gonzalez JMT. 2006a. Occurrence and variability of mycotoxigenic Fusarium species associated to wheat and maize in the South West of Spain. Mycotoxin Res 22: 87-91.

Jurado M, Vazquez C, Marın S et al. 2006b. PCR-Based strategy to detect contamination with mycotoxigenic Fusarium species in maize. Syst Appl Microbiol 29: 681-689.

Jurado M, Vazquez C, Patino B, Gonzalez-Jaen MT. 2005. PCR detection assays for the trichothecene-producing species Fusarium graminearum, Fusarium culmorum, Fusarium poae, Fusarium equiseti and Fusarium sporotrichioides. Syst Appl Microbiol 28: 562568.

Kawakami A, Kato N, Sasaya T et al. 2015. Gibberella ear rot of corn caused by Fusarium asiaticum in Japan. J Gen Plant Pathol 81: 324 327.
Komen JJ, Mutoko CM, Wanyama JM et al. 2008. Economics of postharvest maize grain losses in trans Nzoia and Uasin Gishu Districts of Northwest Kenya. Conference Paper: $12^{\text {th }}$ KARI Biennial Sci. Conf. at Nairobi, Kenya.

Leslie JF, Summerell BA. 2006. The Fusarium Laboratory Manual. Blackwell Publishing, New York.

Li X, Jiang J, Cheng K et al. 2018. First report of leaf spot of Bletilla striata caused by Fusarium asiaticumin China. Plant Dis 102: 17591765 .

Mansour MB, Goh YK, Vujanovic V. 2012. Rapid macroconidia production in Fusarium graminearum 3-and 15-acetyldeoxynivalenol (ADON) chemotypes using sucrose-water medium. Ann Microbiol 62: 965-971.

Mejía D. 2003. MAIZE: Post-Harvest Operation. Food and Agriculture Organization of the United Nations (FAO), Rome.

Mostafa AT, Kazem ZZ. 2011. Fungi associated with harvested corn grains of Golestan province in Iran. Ann Biol Res 2 (5): 681-688.

Muckle AE. 2013. Management of Fusarium Head Blight and Septoria tritici Blotch in Winter Wheat through the use of Host Resistance and Chemical Controls and the Investigation of Fusarium graminearum Chemotype Diversity, Aggressiveness and Toxicity. [Thesis]. University of Guelph, Ontario, Canada.

Mulè G, Susca A, Stea G, Moretti A. 2004. A species-specific PCR assay based on the calmodulin partial gene for identification of Fusarium verticillioides, $F$. proliferatum and F. subglutinans". Eur J Plant Pathol 110: 495-502.

Pakki S. 2016. Mycotoxin contamination, bioecology of Fusarium verticillioides pathogen and its control on maize. J Litbang Pertanian 35 (1): 11-16

Rahjoo V, Zad J, Javan-Nikkhah M, et al. 2008. Morphological and molecular identification of Fusarium isolated from maize ears in Iran. J Plant Pathol. 90 (3): 463-468.

Sampietro DA, Marín P, Iglesias J et al. 2010. A Molecular based strategy for rapid diagnosis of toxigenic Fusarium species associated to cereal grains from Argentina. Fungal Biol 114 (1): 74-81.

Swastika DKS, Kasim F, Suhariyanto K et al. 2004. Maize in Indonesia: Production Systems, Constraints, and Research Priorities. Mexico, D.F. Internaternational Maize and Wheat Improvement Center (CIMMYT), Mexico City.

Tančić S, Stanković S, Lević J, et al. 2012. Diversity of the Fusarium verticillioides and $F$. proliferatum isolates according to their fumonisin B1 production potencial and origin. Genetika 44 (1): 163176.

Vincelli P, Parker G. 2002. Fumonisin, Vomitoxin, and Other Mycotoxins in Corn Produced by Fusarium Fungi. Coop. Ext. Service. Univ. of Kentucky, Coll. of Agric, Kentucky.

Walkowiak S, Owen R, Rodrigue N, Subramaniam R. 2016. Whole genome sequencing and comparative genomics of closely related Fusarium head blight fungi: Fusarium graminearum, F. Meridionale, and $F$. asiaticum. BMC Genomics 17 (1): 1014. DOI: 10.1186/s12864-016-3371-1.

Zhou D, Wang X, Chen G, et al. 2018. The major Fusarium species causing maize ear and kernel rot and their toxigenicity in Chongqing, China. Toxins 10 (2): 90.

Zhu P, Wu L, Liu L et al. 2013. Fusarium asiaticum: an emerging pathogen jeopardizing postharvest Asparagus spears. J Phytopathol 161 (10): 696-703. 